\title{
Symmetry breaking induced by pairwise conversion of neutrinos in compact sources
}

\author{
Shashank Shalgar $\odot^{*}$ and Irene Tamborra $\odot^{\dagger}$ \\ Niels Bohr International Academy \& DARK, Niels Bohr Institute, University of Copenhagen, \\ Blegdamsvej 17, 2100 Copenhagen, Denmark
}

(Received 4 July 2021; accepted 2 February 2022; published 23 February 2022)

\begin{abstract}
A surprising consequence of nonlinear flavor evolution is the spontaneous breaking of the initial symmetries of the neutrino gas propagating in a dense astrophysical environment. We explore the flavor conversion physics by taking into account the polar and azimuthal angular distributions of neutrinos and present the very first example of spontaneous symmetry breaking in the context of fast flavor mixing in the nonlinear regime. Intriguingly, we find that fast flavor mixing does not always develop in the proximity of the angular regions with vanishing electron lepton number, as commonly assumed, and large flavor mixing can rapidly spread through all neutrino modes. Such behavior cannot be predicted from the linear regime of the flavor evolution. These results can have major consequences on the physics of compact astrophysical objects.
\end{abstract}

DOI: 10.1103/PhysRevD.105.043018

\section{INTRODUCTION}

In neutrino dense environments, the flavor evolution of neutrinos is affected by the interaction of neutrinos among themselves [1-4]. The nonlinear feedback in the neutrino equations of motion is too complicated to be understood analytically, and not enough progress has been made on the numerical front. A recent development concerns the possibility that fast pairwise conversion occurs [3-7]. In the case of ordinary interactions of neutrinos with matter, leading to the well known Mikheev-Smirnov-Wolfenstein effect [8-10], flavor mixing depends on the energy of (anti) neutrinos. Fast pairwise conversion is instead driven by the angular distributions of (anti)neutrinos [5-7,11-13]; however, the dependence of neutrino flavor evolution on neutrino energy cannot be ignored in the nonlinear phase $[14,15]$.

The angular distribution of (anti)neutrinos is characterized by two independent angular variables, polar and azimuthal. Up to now, for the sake of simplicity, the azimuthal distribution of (anti)neutrinos has been neglected in most cases under the assumption of azimuthal symmetry; we refer the interested reader to, e.g., Refs. [11,16,17] for preliminary work incorporating the azimuthal variable in the linear phase of neutrino pairwise conversion. In the

\footnotetext{
shashank.shalgar@nbi.ku.dk

tamborra@nbi.ku.dk
}

Published by the American Physical Society under the terms of the Creative Commons Attribution 4.0 International license. Further distribution of this work must maintain attribution to the author(s) and the published article's title, journal citation, and DOI. Funded by SCOAP ${ }^{3}$. azimuthally symmetric framework, it has been shown that fast flavor instabilities develop in the proximity of crossings in the polar angular distribution of the electron neutrino lepton number (ELN) [4,12,18-21]. The existence of such flavor instability depends on the steepness and the depth of the ELN crossing $[19,20]$ and may be affected by collisions with the medium [22-26]. However, despite the existence of ELN crossings, significant fast flavor mixing may not be achieved [27], and the ELN crossings could be affected by neutrino advection dynamically [28].

In this paper, we focus on angular distributions that are not azimuthally symmetric and investigate the flavor conversion physics. Azimuthal angular distributions could also be instrumental in determining the existence of spontaneous symmetry breaking. The latter is a peculiar feature of collective flavor evolution discovered in the context of slow neutrino self-interaction [29-31], but never explored for fast flavor conversion.

We carry out numerical simulations of the fast flavor evolution in three flavors, including polar and azimuthal angular modes. For the first time, we show that spontaneous symmetry breaking may significantly impact the fast flavor conversion rate in the nonlinear regime. Moreover, while fast flavor evolution in the nonlinear regime in an azimuthally symmetric system seems to imply that flavor conversion develops in the vicinity of the ELN crossings [4], this correlation is no longer valid for certain configurations of the zenith and azimuthal angular modes. Hence, contrary to common assumptions in the literature, the correlation between the location of the ELN crossing and the onset of flavor evolution is a special case observed in azimuthally symmetric configurations. 


\section{NEUTRINO ANGULAR DISTRIBUTIONS}

At each point in space and time, a homogeneous neutrino field can be expressed in terms of angular distributions that are functions of the polar and azimuthal angles, $\theta$ and $\phi$. Assuming that the (anti)neutrinos are monoenergetic, for each $(\theta, \phi)$, the flavor information can be encoded in a $3 \times 3$ density matrix for (anti)neutrinos, $\rho(\bar{\rho})$, whose diagonal elements denote the occupation number of (anti)neutrinos for each flavor.

When only the angular distribution in $\theta$ is considered, a necessary condition for fast flavor instabilities is the occurrence of at least one ELN crossing [12,21,32]. When the azimuthal angular distribution is also taken into account, the ELN crossing occurs for a locus of points which can be plotted on a two dimensional surface, e.g., using the Mollweide projection. We classify the angular distribution through the number of disconnected rings with vanishing ELN (instead of the number of ELN crossings usually adopted in azimuthally symmetric systems); e.g., an azimuthally symmetric distribution with one crossing would thus correspond to one ring of vanishing ELN, two crossings would correspond to two ELN rings, and so on.

To investigate the qualitative differences arising in the flavor conversion physics, we consider a set of representative configurations of the angular distributions for $\nu_{e}$ 's and $\bar{\nu}_{e}$ 's (Cases A, B, C, and D), two with one ELN ring and two with two ELN rings, respectively

$$
\begin{gathered}
\rho_{e e, \mathrm{~A}, \mathrm{~B}, \mathrm{C}, \mathrm{D}}=\frac{1}{4 \pi}, \\
\bar{\rho}_{e e, \mathrm{~A}}=\frac{1}{2 \pi}\left\{0.47+0.05 \exp \left[-(\cos \theta-1)^{2}\right]\right\}, \\
\bar{\rho}_{e e, \mathrm{~B}}=\frac{1}{2 \pi}\left\{0.47+0.05\left[\exp \left(-2(\theta-\pi / 2)^{2}\right) \exp \left(-0.5(\phi-\pi)^{2}\right)\right]\right\}, \\
\bar{\rho}_{e e, \mathrm{C}}=\frac{1}{2 \pi}\left\{0.48+0.05\left[\exp \left(-2.5(\theta-\pi / 2)^{2}\right) \exp \left(-2(\phi-\pi)^{2}\right)+\exp \left(-2(\theta-\pi / 2)^{2}\right) \exp \left(-2(\phi-\pi)^{2}\right)\right]\right\}, \\
\bar{\rho}_{e e, \mathrm{D}}=\frac{1}{2 \pi}\left\{0.49+0.05\left[\exp \left(-2.5(\theta-\pi / 2)^{2}\right) \exp \left(-2(\phi-\pi)^{2}\right)+\exp \left(-2(\theta-\pi / 2)^{2}\right) \exp \left(-2(\phi-\pi)^{2}\right)\right]\right\} .
\end{gathered}
$$

We assume that the nonelectron type neutrinos are initially negligible and are generated through flavor mixing.

In order to quantify the differences among the angular distributions of Cases A-D, we introduce the parameters

$$
\begin{gathered}
\operatorname{ELN} \uparrow= \begin{cases}\frac{\int\left(\rho_{e e}-\bar{\rho}_{e e}\right) d \cos \theta d \phi}{\int\left(\rho_{e e}+\bar{\rho}_{e e}\right) d \cos \theta d \phi} & \text { for } \rho_{e e} \geq \bar{\rho}_{e e} \\
0 & \text { otherwise, }\end{cases} \\
\operatorname{ELN} \downarrow= \begin{cases}\frac{\int\left(\bar{\rho}_{e e}-\rho_{e e}\right) d \cos \theta d \phi}{\int\left(\rho_{e e}+\bar{\rho}_{e e}\right) d \cos \theta d \phi} & \text { for } \rho_{e e} \leq \bar{\rho}_{e e} \\
0 & \text { otherwise. }\end{cases}
\end{gathered}
$$

The parameters ELN $\uparrow$ and ELN $\downarrow$ help to distinguish among configurations with similar total ELN, but overall different angular distributions. For Cases A-D, the values of ELN $\uparrow$ and ELN $\downarrow$ are reported in Table I to illustrate the relative excess of $\rho_{e e}$ or $\bar{\rho}_{e e}$ in the two regions separated by vanishing ELN, together with the difference between ELN $\uparrow$ and ELN $\downarrow$ which gives the overall excess of $\nu_{e}$ over $\bar{\nu}_{e}$ normalized to the total number of $\nu_{e}$ and $\bar{\nu}_{e}$. From Table I, we can clearly see that Cases $\mathrm{A}$ and $\mathrm{C}$ have an excess of $\nu_{e}$ over $\bar{\nu}_{e}$, while Cases B and D have a very small total ELN number (with respect to Cases A and C) and an excess of $\bar{\nu}_{e}$ over $\nu_{e}$. It is worth highlighting that Cases B and $\mathrm{D}$ do not have similar distributions of $\nu_{e}$ and $\bar{\nu}_{e}$, although they both have a small total ELN, as shown by the values of ELN $\uparrow$ and ELN $\downarrow$ in Table I.

To better appreciate the differences among Cases A-D, the angular distribution of $\rho_{e e}-\bar{\rho}_{e e}$ is shown for all configurations in the left panels of Fig. 1. Cases A and $\mathrm{B}$ have one single ELN ring, but Case A shows one crossing in $\theta$ and it is azimuthally symmetric (i.e., this

TABLE I. Parameters characterizing the initial angular distributions for Cases A-D. The second and third columns represent ELN $\uparrow$ and ELN $\downarrow$ (Eqs. (6) and (7), respectively. The fourth column is the difference between the first two columns and is indicative of the commonly adopted total ELN number. The last column tabulates the number of ELN rings.

\begin{tabular}{lcccc}
\hline \hline & ELN $\uparrow$ & ELN $\downarrow$ & ELN $\uparrow-E L N \downarrow$ & $\#$ of rings \\
\hline Case A & 0.01257 & 0.00456 & 0.00801 & 1 \\
Case B & 0.00527 & 0.00529 & -0.00002 & 1 \\
Case C & 0.01254 & 0.00256 & 0.00998 & 2 \\
Case D & 0.00499 & 0.00510 & -0.00012 & 2 \\
\hline \hline
\end{tabular}



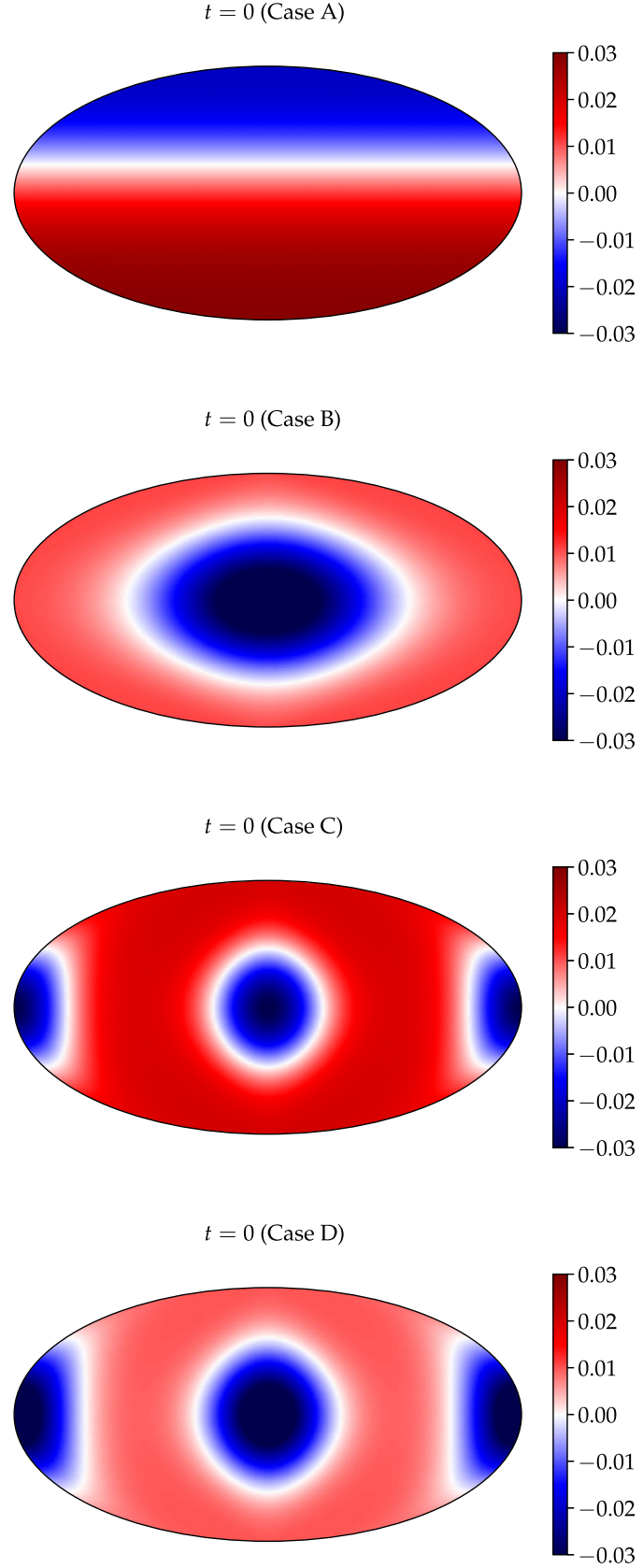
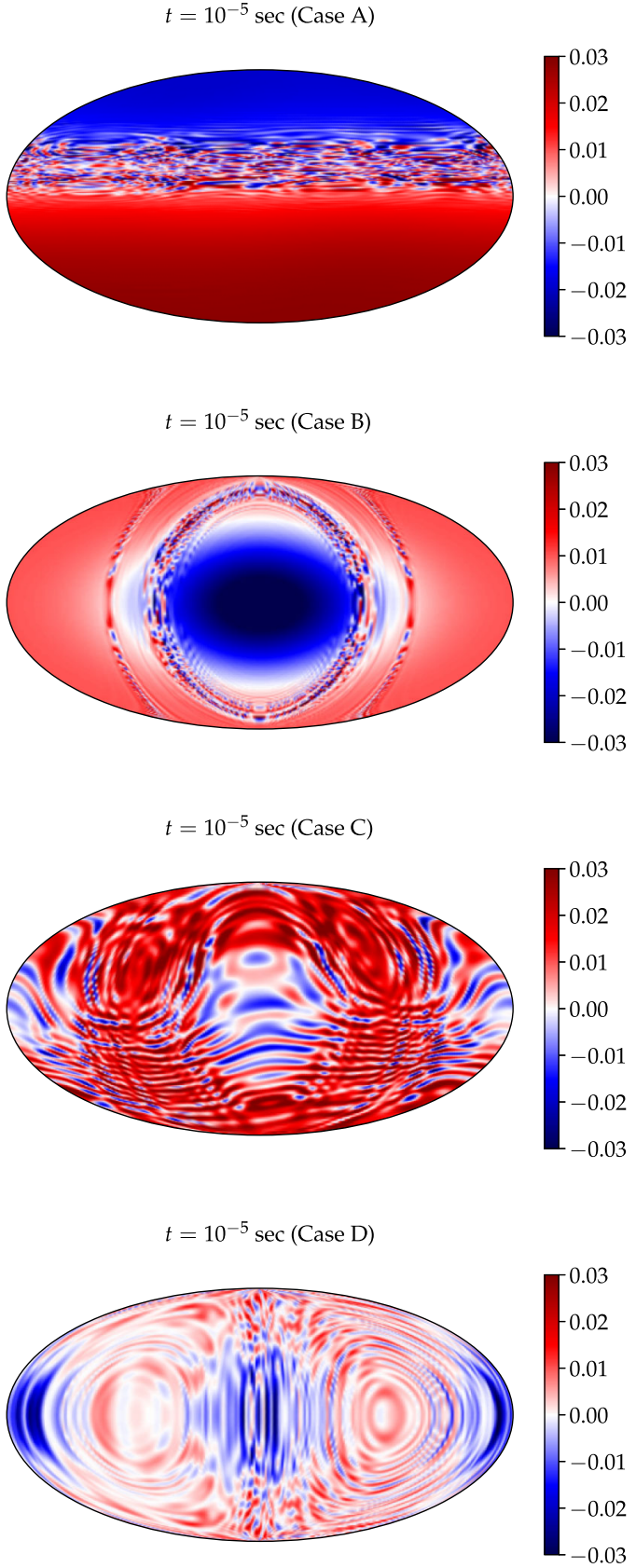

FIG. 1. Left panels: Mollweide projections of the angular distribution of the initial $\rho_{e e}-\bar{\rho}_{e e}$ for Cases A-D from top to bottom, respectively (see Eqs. (2)-(5)). On each skymap, the polar variable $\theta$ varies from 0 at the South pole to $\pi$ at the North pole; while the azimuthal variable $\phi$ runs from 0 on the left-hand side of the map to $2 \pi$ on the right-hand side. The white region in each map shows the vanishing ELN ring. Right panels: Mollweide projections of the angular distribution of the final $\rho_{e e}-\bar{\rho}_{e e}$ at $t=10^{-5}$ sec. Spontaneous symmetry breaking occurs for all cases and it is especially evident in Case D; the onset of flavor mixing does not always follow the ELN rings, as clearly evident in Cases B-D.

configuration can be directly mapped onto a one-dimensional angular distribution similar to the ones commonly adopted in the literature). Despite the different orientation of their respective ELN ring, Cases A and B have been selected for a direct comparison with the existing literature, where it has been shown that the instability type depends on the kind of ELN crossing and the flavor outcome is strictly related to the width and height of the ELN crossing $[4,19,20]$.

Cases $C$ and D have two ELN rings, with Case D having larger rings than Case $\mathrm{C}$ (see also Table I). In the limit of azimuthal symmetry, it was found that distributions with two ELN crossings are not necessarily unstable [32,33]. However, as we show in the following, the magnitude of the 
flavor instability in the linear regime is not an indication of the amount of flavor conversion, as also found in other investigations of fast flavor conversion in the nonlinear regime, see e.g., $[14,15]$.

It is also worth noticing that Cases A-D involve symmetries in the initial conditions (see left panels of Fig. 1); for example, there is a reflection symmetry around $\phi=\pi$ and for all cases, except for Case A, there is a reflection symmetry around $\theta=\pi / 2$. However, as shown in the following, these initial symmetries are broken through flavor mixing.

\section{EQUATIONS OF MOTION}

The evolution of neutrinos and antineutrinos is governed by the Heisenberg equations

$$
i \frac{d \rho}{d t}=[H, \rho] \quad \text { and } \quad i \frac{d \bar{\rho}}{d t}=[\bar{H}, \bar{\rho}] ;
$$

and the Hamiltonian includes three components corresponding to the vacuum, matter, and self-interaction terms

$$
\begin{gathered}
H=H_{\mathrm{vac}}+H_{\mathrm{mat}}+H_{\nu \nu}, \\
\bar{H}=-H_{\mathrm{vac}}+H_{\mathrm{mat}}+H_{\nu \nu} .
\end{gathered}
$$

The three terms of the Hamiltonian are defined as

$$
\begin{gathered}
H_{\text {vac }}=\frac{1}{2 E} U \operatorname{diag}\left(0, \delta m^{2}, \Delta m^{2}\right) U^{\dagger}, \\
H_{\text {mat }}=\operatorname{diag}\left(\sqrt{2} G_{\mathrm{F}} n_{e}, 0,0\right), \\
H_{\nu \nu}=\mu \int(\rho-\bar{\rho})\left(1-v \cdot v^{\prime}\right) d^{3} v^{\prime} .
\end{gathered}
$$

Here $U\left(\theta_{12}, \theta_{13}, \theta_{23}\right)$ is the $3 \times 3$ Pontecorvo-MakiNakagawa-Sakata matrix expressed as a function of the three mixing angles, $\delta m^{2}$ and $\Delta m^{2}$ are the mass-squared differences of neutrinos, $G_{\mathrm{F}}$ is the Fermi constant, $n_{e}$ is the effective number density of electrons, $\mu$ is the strength of neutrino self-interaction, $v$ is the velocity of the neutrinos under consideration and $v^{\prime}$ represents the velocity of the neutrinos in the medium.

The $\left(1-v \cdot v^{\prime}\right)$ term in $H_{\nu \nu}$ can be rewritten in terms of $\theta$ and $\phi$ explicitly using,

$v \cdot v^{\prime}=\cos \theta \cos \theta^{\prime}+\sin \theta \sin \theta^{\prime}\left(\cos \phi \cos \phi^{\prime}+\sin \phi \sin \phi^{\prime}\right)$.

It is worth noticing that since the inner product in $H_{\nu \nu}$ is not symmetric in $\theta$ and $\phi$, the flavor evolution cannot be decomposed into polar and azimuthal modes; nevertheless, it is possible to rotate the location of the ELN rings across the surface spanned by the polar and azimuthal angles. Moreover, the term $v \cdot v^{\prime}$ in the Hamiltonian highlights the occurrence of symmetries in $H_{\nu \nu}$ that are not necessarily the same as the ones of the ELN rings. By taking into account Eq. (14), $H_{\nu \nu}$ can be split in four terms, that are helpful in identifying the symmetries of the system as further discussed in the following:

$H_{\nu \nu}=h_{0}+h_{1} \sin \theta \cos \phi+h_{2} \sin \theta \sin \phi+h_{3} \cos \theta ;$

e.g., if $h_{3}$ is initially zero, this is an indication of a discrete reflection symmetry of the Hamiltonian across $\theta=\pi / 2$; while the dynamical generation of $h_{3}$ can only be a result of spontaneous breaking of this discrete symmetry.

In the numerical simulations, we assume $\delta m^{2}=$ $7.53 \times 10^{-5} \mathrm{eV}^{2}, \quad \Delta m^{2}=2.453 \times 10^{-3} \mathrm{eV}^{2} \quad$ [34], $\quad \mu=$ $2.5 \times 10^{4} \mathrm{~km}^{-1}$, and only consider one energy mode $E=50 \mathrm{MeV}$. We ignore the matter term and compensate for that by using $\theta_{12}=\theta_{13}=\theta_{23}=10^{-3}$ rad (this approximation has been shown to hold in the three flavor case [15]).

We perform the numerical simulations by discretizing the angular distribution over a grid of $200 \times 200$ bins, which is uniform over $\cos \theta$ and $\phi$; note that, while this grid choice samples the polar region more densely, the equatorial region is not sparsely sampled. For Case A, we have also performed the computation using $500 \times 500$ bins to ensure that numerical convergence is achieved (results not shown here). In the numerical solution of the neutrino equations of motion, the seed to trigger the symmetry breaking modes is not put by hand, but it is provided by the inaccuracy in the computation of the trigonometric functions, which do not exactly satisfy the condition $\sin (\phi)+\sin (-\phi)=0$. For completeness, the evolution of the angle-averaged $\nu_{e}$ flavor content is reported in Appendix.

\section{FLAVOR EVOLUTION IN THE NONLINEAR REGIME}

The top right panel of Fig. 1 shows the map of $\rho_{e e}-\bar{\rho}_{e e}$ of Case A at $t=10^{-5} \mathrm{sec}$. Although we start with a system that has azimuthal symmetry, the initial reflection symmetry around $\phi=\pi$ is broken spontaneously (see also the animations in the supplemental material [35]). The symmetry breaking is particularly visible in the top panel of Fig. 2, showing the Mollweide projection of $P_{e e}$, where the latter is defined as the ratio between the final and initial content of $\nu_{e}$,

$$
P_{e e}=\frac{\rho_{e e}(\cos \theta, \phi, t)}{\rho_{e e}(\cos \theta, \phi, t=0)}
$$

there are regions across the Mollweide map where near maximal flavor transformation is achieved (see Appendix for additional details). The right top panel of Fig. 1 and the top panel of Fig. 2 suggest that the onset of the flavor evolution for Case A starts in the proximity of the ELN 
ring, which happens to be circular in shape. This finding is in agreement with work on azimuthally symmetric cases $[4,19,20]$. Flavor mixing then spreads in the upper hemisphere in the nonlinear regime.

Case B is another configuration with one ELN ring (second panel on the left of Fig. 1). As shown in the corresponding panel on the right of Fig. 1 and in the second panel of Fig. 2 (as well as in the animations in the
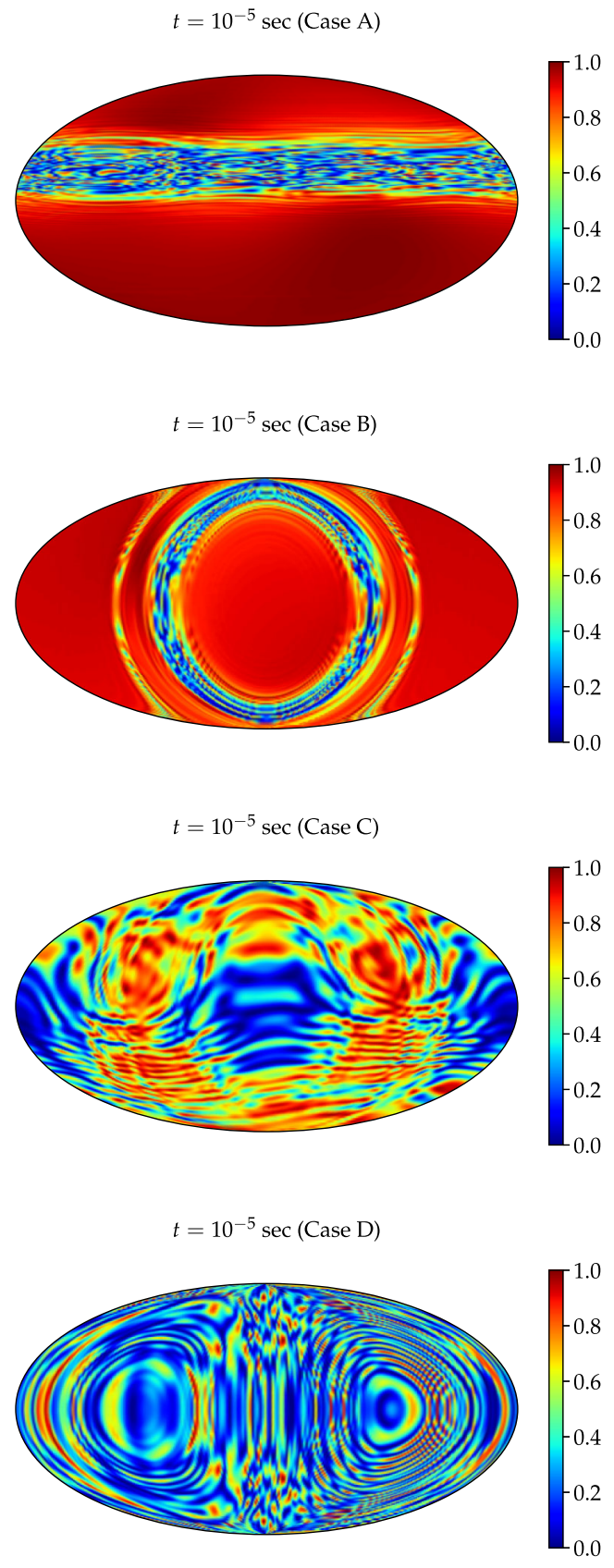

FIG. 2. Mollweide projections of the ratio between the initial and final content of $\nu_{e}$ [defined as in Eq. (15)] at $t=10^{-5} \mathrm{sec}$ for Cases A-D from top to bottom, respectively. Maximal flavor mixing is achieved for all Cases for certain regions across the Mollweide map. supplemental material [35]), the onset of flavor evolution occurs along a region that is circular in shape, which only intersects the initial ELN ring, while not being aligned with it. This result has been overlooked in azimuthally symmetric configurations (see, e.g., Case A for comparison). The reason for such a trend can be easily understood by looking at the Hamiltonian of the system [Eqs. (13) and (14)]; the Hamiltonian is only related to the ELN angular distribution through the integration over the polar and azimuthal angles, hence $H_{\nu \nu}$ can have symmetries that the initial ELN distribution does not have; in Case $\mathrm{B}, h_{1} \neq 0$ and $h_{1} \gg h_{0,2,3} \simeq 0$ at $t=0$ - then $h_{1}$ reaches the nonlinear regime followed by $h_{0}$ and dominates the onset of the nonlinear phase (see Appendix for more details). As a consequence, flavor mixing develops along a circle, but does not track the ELN ring.

The phenomenology of fast flavor conversion becomes even more interesting when two ELN rings are considered (Cases C and D). In fact, even if the two initial configurations appear to be very similar except for the overall opposite ELN sign (see Table I and the two panels on the bottom left of Fig. 1), the final $\rho_{e e}-\bar{\rho}_{e e}$ configurations are very different in the two panels on the bottom right of Fig. 1. For Case $\mathrm{C}$, the onset of flavor mixing occurs along one ring crossing the North and the South poles of the map of angular distribution (see animations in the supplemental material [35]), then the flavor evolution is such that the final flavor configuration mixing happens to resemble the shape of the initial ELN rings.

In Case D, the ELN pattern after flavor mixing is completely different with respect to the initial ELN configuration and the angular regions with significant flavor evolution are not correlated in an obvious manner with the regions where the ELN rings are initially located, as is also visible from the animations in the supplemental material [35]. In addition, it is clear from the bottom panel of Fig. 2 that full flavor conversion takes place across the whole angular range in Case D. The difference in the outcome of the flavor evolution is also seen in the evolution of the angle averaged survival probability in Appendix. The strong symmetry-breaking effects observable in Case D are not determined by the small magnitude of the ELN per sethe latter has indeed been employed in various sets of initial configurations, see e.g., Refs. [27,36]—it is also not determined by the negative sign of the total ELN, in fact we have explored other nonazimuthally symmetric configurations with overall negative ELN without finding dramatic spontaneous symmetry breaking effects (results not shown here).

It is important to stress that such symmetry breaking is different in nature with respect to the one observed in the context of slow neutrino self-interaction [29-31], where the of azimuthal symmetry breaking has been investigated in connection to breaking of spatial symmetry. Fast flavor evolution can occur over small length scales and the region 
over which flavor mixing occurs can be approximated to have homogeneous initial conditions. Slow neutrino selfinteractions, on the other hand, occur over larger length scales and have been investigated mostly in the context of neutrino-bulb model; as such, the initial conditions are not homogeneous resulting in a system that cannot be solved in a self-consistent manner if we only break the azimuthal symmetry while preserving the initial spatial symmetry. In the case of fast flavor mixing, the angular symmetry breaking is purely driven by the angular distributions of neutrinos. The symmetry breaking becomes fully evident in the nonlinear regime, emphasizing the need for going beyond the linear stability analysis in the context of fast flavor mixing in agreement with Refs. [14,15,22,37-39].

\section{CONCLUSIONS}

In this paper, for the first time, we explore the flavor conversion physics in three flavors and by including the polar and azimuthal angular distributions. Under the assumption of azimuthal symmetry, it is usually believed that fast flavor conversion develops in the angular bins in the proximity of the electron lepton number ring.

When the assumption of azimuthal symmetry is relaxed, we demonstrate that the onset of flavor mixing does not follow the ELN rings; flavor mixing develops along angular regions that are circular in shape and that intersect the ELN ring, but do not always fully overlap with the ELN ring. This is an interesting feature of fast conversion. The correlation between the location of the ELN ring and the one of the onset of flavor evolution in the azimuthally symmetric configurations seems to be a peculiar feature of these configurations. This phenomenon is due to the fact that the $\nu-\nu$ Hamiltonian can be azimuthally symmetric, even if the ELN distribution is not.

As flavor evolution enters the nonlinear regime, the angular regions affected by mixing are not restricted to the initial circular region; all the symmetries present in the initial ELN configuration are broken. In the examples investigated in this work, even the discrete reflection symmetry imposed in the initial configuration of our system is spontaneously broken by the floating-point error in the numerical simulation.

Intriguingly, we find configurations for which the angular distribution after flavor conversion is entirely different from the initial one and flavor mixing affects all angular modes. This effect does not manifest in the context of slow self-interactions and it is observed to occur for fast flavor mixing for the first time. These findings reinforce the need for more substantial work on the fast flavor evolution in the nonlinear regime.

We focused on the flavor evolution in the case of a spatially homogeneous neutrino gas; however, as the symmetry breaking in the neutrino flavor conversion starts manifesting in the nonlinear regime, the assumption of homogeneity may eventually play an important role. In addition, we have ignored the effect of direction changing collisions on the system, which could further aid the symmetry breaking effects. Our work highlights that it is necessary to carry out a thorough investigation of the physics linked to spontaneous symmetry breaking due to flavor mixing within realistic astrophysical systems and in the nonlinear regime.

\section{ACKNOWLEDGMENTS}

We are grateful to the Villum Foundation (Project No. 13164), the Danmarks Frie Forskningsfonds (Project No. 8049-00038B), the MERAC Foundation, and the Deutsche Forschungsgemeinschaft through Sonderforschungbereich SFB 1258 "Neutrinos and Dark Matter in Astro- and Particle Physics" (NDM).

\section{APPENDIX: HIGHLIGHTS ON THE DEVELOPMENT OF SYMMETRY BREAKING}

In order to quantify the overall amount of flavor mixing for Cases A-D, Fig. 3 shows the temporal evolution

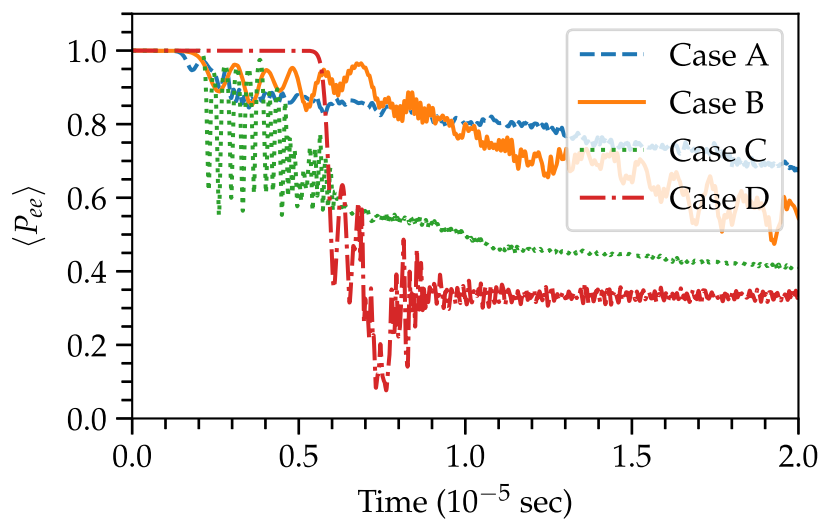

FIG. 3. Angle averaged ratio between the initial and final content of $\nu_{e}$ as a function of time for Cases A, B, C, and D. For certain initial configurations, large flavor mixing is achieved.

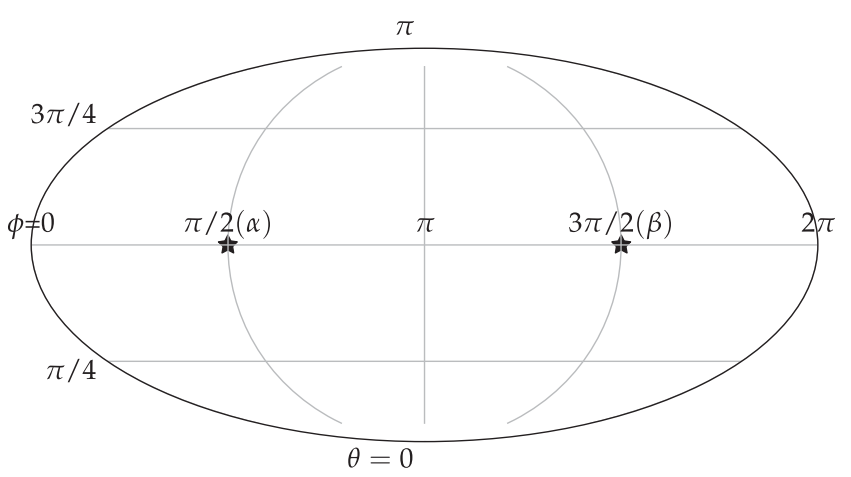

FIG. 4. Mollweide map illustrating the polar angle $(\theta)$ and the azimuthal one $(\phi)$ adopted to characterize the angular distributions of (anti)neutrinos. The benchmark points $\alpha$ and $\beta$, marked by a star, are adopted as representative cases to investigate the breaking of reflection symmetry around $(\theta=\pi / 2, \phi=\pi)$. 


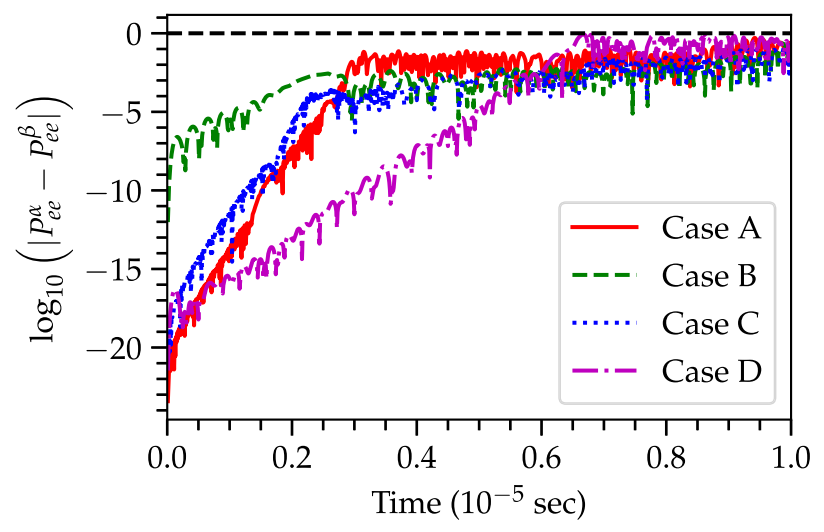

FIG. 5. Temporal evolution of the difference in the ratio between the initial and final content of $\nu_{e}$ for the points $\alpha$ and $\beta$ marked in Fig. 4 for Cases A-D. In all cases, the initial reflection symmetry around $(\theta=\pi / 2, \phi=\pi)$ is broken through mixing.

of the angle averaged ratio between the initial and final content of $\nu_{e} ;\left\langle P_{e e}\right\rangle=\left[\int \rho_{e e}(\cos \theta, \phi, t) d \cos \theta d \phi\right] /$ $\left[\int \rho_{e e}(\cos \theta, \phi, t=0) d \cos \theta d \phi\right]$. A qualitative difference in the flavor evolution of Cases $\mathrm{A}$ and $\mathrm{B}$, as well as $\mathrm{C}$ and D, can be seen in Fig. 3 despite the very similar angular distributions. In particular, large flavor mixing can be achieved for Cases C and D.

In this appendix we intend to provide details on the impact of the symmetry breaking on the flavor evolution. To this purpose, we focus on two angular locations, $\alpha \equiv$ $(\theta=\pi / 2, \phi=\pi / 2)$ and $\beta \equiv(\theta=\pi / 2, \phi=3 \pi / 2)$, displayed in Fig. 4 that initially have the same density matrix due to the reflection symmetry with respect to $\phi=\pi$.

The deviation of the $\nu_{e}$ survival probabilities between $\alpha$ and $\beta$ is a clear indicator of the symmetry breaking and is displayed in Fig. 5. A close look at all cases shown in Fig. 5 highlights that the linear regime does not give an indication of the magnitude of symmetry breaking that can be expected in the nonlinear regime and the time at which such symmetry breaking would manifest.

In all cases, the reflection symmetry around $\phi=\pi$ is broken (see also the animations). For Cases A and B, the reflection symmetry is broken early in the linear regime, but the exponential growth is halted before it reaches magnitudes of order unity. For Case $\mathrm{C}$, although the reflection symmetry is maintained in the initial stages of the nonlinear regime, the symmetry is slowly broken on a small angular
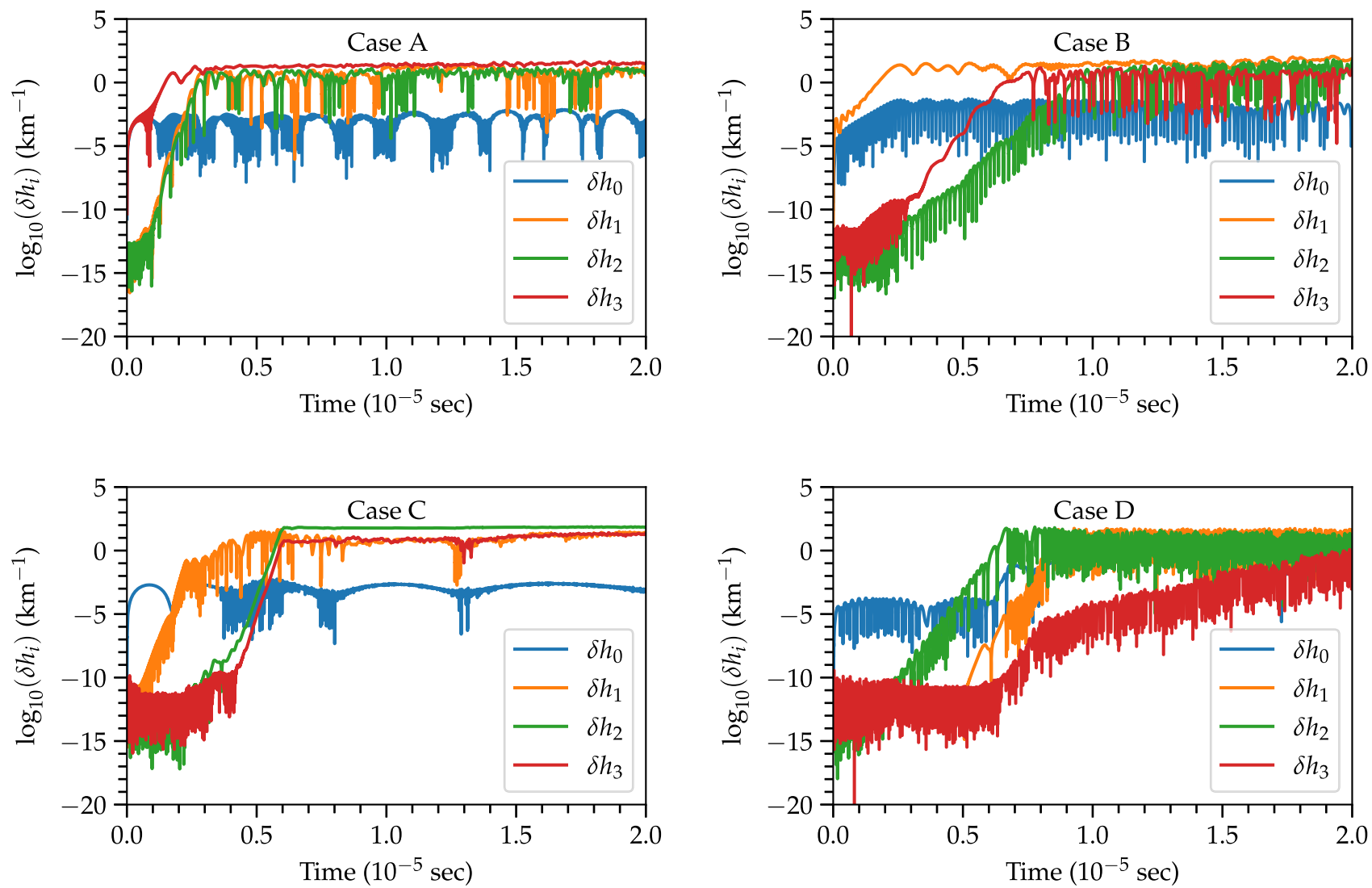

FIG. 6. Temporal evolution of various components of the Hamiltonian as defined in Eq. (14) for Cases A-D from top left to bottom right, respectively. The $y$-axis shows the deviation of the components of the Hamiltonian from their initial value at $t=0$. For Case A (B), $h_{3}\left(h_{1}\right)$ is the dominant component initially; for Case C (D), $h_{0}$ dominates at the initial time, however $h_{1}\left(h_{2}\right)$ evolves the most during the initial times quickly reaching the nonlinear regime. 
scale initially; then, it spreads to all angular scales, as visible from Fig. 5. Case D is qualitatively very different in that, as soon as the nonlinear regime begins, there is a dramatic breaking of the reflection symmetry and the exponential growth of the difference between $P_{e e}^{\alpha}$ and $P_{e e}^{\beta}$ continues to grow until it reaches magnitudes of order unity.

The evolution of the various components of the Hamiltonian defined as in Eq. (14) and displayed in Fig. 6 reinforces the crucial role of nonlinearity in the symmetry breaking, and the fact that linear stability analysis cannot give us an indication of the symmetries of the region along which the onset of flavor evolution occurs. For example, the evolution of $H_{\nu \nu}$ for Case D shows that the most dominant component of the Hamiltonian is the one which is independent of the angle $\left(h_{0}\right)$ at first, but the onset of flavor evolution occurs predominant along a circular region defined by $\phi=$ $0, \pi$ (see also the animations). The circular region where the onset of flavor mixing is located has the same symmetries as the $h_{3}$ term of the Hamiltonian.

We have performed a calculation with several different angular distributions (results not shown here) and there are examples in which $h_{3}$ dominates the onset of the nonlinear regime even though the $h_{2}$ term is the largest at the initial time. The symmetry or lack thereof is thus determined by a nontrivial combination of growth rates in the linear regime and the initial values of various terms of the Hamiltonian, together with the initial ELN configuration and the conservation laws fulfilled by our system.

These findings are not an artifact of the three-flavor calculations, but are exclusively dependent on the initial configurations of the system. The same trend is visible in the two flavor scenario (see the animations); the differences between the two- and three-flavor scenarios that we find for all cases including the polar and azimuthal angular variables are comparable to the ones discussed in Ref. [15].
[1] H. Duan, G. M. Fuller, and Y.-Z. Qian, Collective neutrino oscillations, Annu. Rev. Nucl. Part. Sci. 60, 569 (2010).

[2] A. Mirizzi, I. Tamborra, H.-T. Janka, N. Saviano, K. Scholberg, R. Bollig, L. Hüdepohl, and S. Chakraborty, Supernova neutrinos: Production, oscillations and detection, Riv. Nuovo Cimento 39, 1 (2016).

[3] S. Chakraborty, R. Hansen, I. Izaguirre, and G. G. Raffelt, Collective neutrino flavor conversion: Recent developments, Nucl. Phys. B908, 366 (2016).

[4] I. Tamborra and S. Shalgar, New developments in flavor evolution of a dense neutrino gas, Annu. Rev. Nucl. Part. Sci. 71, 165 (2021).

[5] R. F. Sawyer, Neutrino Cloud Instabilities Just above the Neutrino Sphere of a Supernova, Phys. Rev. Lett. 116, 081101 (2016).

[6] R. F. Sawyer, The multi-angle instability in dense neutrino systems, Phys. Rev. D 79, 105003 (2009).

[7] R. F. Sawyer, Speed-up of neutrino transformations in a supernova environment, Phys. Rev. D 72, 045003 (2005).

[8] S. P. Mikheev and A. Yu. Smirnov, Neutrino oscillations in a variable density medium and neutrino bursts due to the gravitational collapse of stars, Sov. Phys. JETP 64, 4 (1986), https://inspirehep.net/literature/240717.

[9] S. P. Mikheyev and A. Yu. Smirnov, Resonance enhancement of oscillations in matter and solar neutrino spectroscopy, Yad. Fiz. 42, 1441 (1985), https://inspirehep.net/ literature/228623.

[10] L. Wolfenstein, Neutrino oscillations in matter, Phys. Rev. D 17, 2369 (1978).

[11] S. Chakraborty, R. S. Hansen, I. Izaguirre, and G. G. Raffelt, Self-induced neutrino flavor conversion without flavor mixing, J. Cosmol. Astropart. Phys. 03 (2016) 042.
[12] I. Izaguirre, G. G. Raffelt, and I. Tamborra, Fast Pairwise Conversion of Supernova Neutrinos: A Dispersion-Relation Approach, Phys. Rev. Lett. 118, 021101 (2017).

[13] S. Airen, F. Capozzi, S. Chakraborty, B. Dasgupta, G. G. Raffelt, and T. Stirner, Normal-mode analysis for collective neutrino oscillations, J. Cosmol. Astropart. Phys. 12 (2018) 019 .

[14] S. Shalgar and I. Tamborra, Dispelling a myth on dense neutrino media: Fast pairwise conversions depend on energy, J. Cosmol. Astropart. Phys. 01 (2021) 014.

[15] S. Shalgar and I. Tamborra, Three flavor revolution in fast pairwise neutrino conversion, Phys. Rev. D 104, 023011 (2021).

[16] M. D. Azari, S. Yamada, T. Morinaga, W. Iwakami, H. Okawa, H. Nagakura, and K. Sumiyoshi, Linear analysis of fast-pairwise collective neutrino oscillations in corecollapse supernovae based on the results of Boltzmann simulations, Phys. Rev. D 99, 103011 (2019).

[17] H. Nagakura, T. Morinaga, C. Kato, and S. Yamada, Fastpairwise collective neutrino oscillations associated with asymmetric neutrino emissions in core-collapse supernovae, Astrophys. J. 886, 139 (2019).

[18] B. Dasgupta, A. Mirizzi, and M. Sen, Fast neutrino flavor conversions near the supernova core with realistic flavordependent angular distributions, J. Cosmol. Astropart. Phys. 02 (2017) 019.

[19] C. Yi, L. Ma, J. D. Martin, and H. Duan, Dispersion relation of the fast neutrino oscillation wave, Phys. Rev. D 99, 063005 (2019).

[20] J. D. Martin, C. Yi, and H. Duan, Dynamic fast flavor oscillation waves in dense neutrino gases, Phys. Lett. B 800, 135088 (2020).

[21] T. Morinaga, Fast neutrino flavor instability and neutrino flavor lepton number crossings, arXiv:2103.15267. 
[22] S. Shalgar and I. Tamborra, A change of direction in pairwise neutrino conversion physics: The effect of collisions, Phys. Rev. D 103, 063002 (2021).

[23] F. Capozzi, B. Dasgupta, A. Mirizzi, M. Sen, and G. Sigl, Collisional Triggering of Fast Flavor Conversions of Supernova Neutrinos, Phys. Rev. Lett. 122, 091101 (2019).

[24] S. Shalgar and I. Tamborra, On the occurrence of crossings between the angular distributions of electron neutrinos and antineutrinos in the supernova core, Astrophys. J. 883, 80 (2019).

[25] L. Johns, Collisional flavor instabilities of supernova neutrinos, arXiv:2104.11369.

[26] J. D. Martin, J. Carlson, V. Cirigliano, and H. Duan, Fast flavor oscillations in dense neutrino media with collisions, Phys. Rev. D 103, 063001 (2021).

[27] I. Padilla-Gay, S. Shalgar, and I. Tamborra, Multidimensional solution of fast neutrino conversions in binary neutron star merger remnants, J. Cosmol. Astropart. Phys. 01 (2021) 017.

[28] S. Shalgar, I. Padilla-Gay, and I. Tamborra, Neutrino propagation hinders fast pairwise flavor conversions, J. Cosmol. Astropart. Phys. 06 (2020) 048.

[29] G. G. Raffelt, S. Sarikas, and D. de Sousa Seixas, Axial Symmetry Breaking in Self-Induced Flavor Conversion of Supernova Neutrino Fluxes, Phys. Rev. Lett. 111, 091101 (2013); Erratum, Phys. Rev. Lett. 113, 239903 (2014).
[30] H. Duan and S. Shalgar, Flavor instabilities in the neutrino line model, Phys. Lett. B 747, 139 (2015).

[31] S. Abbar, H. Duan, and S. Shalgar, Flavor instabilities in the multiangle neutrino line model, Phys. Rev. D 92, 065019 (2015).

[32] F. Capozzi, G. Raffelt, and T. Stirner, Fast neutrino flavor conversion: Collective motion vs decoherence, J. Cosmol. Astropart. Phys. 09 (2019) 002.

[33] S. Bhattacharyya and B. Dasgupta, Fast flavor oscillations of astrophysical neutrinos with $1,2, \ldots, \infty$ crossings, J. Cosmol. Astropart. Phys. 07 (2021) 023.

[34] P. A. Zyla et al. (Particle Data Group), Review of particle physics, Prog. Theor. Exp. Phys. (2020), $083 \mathrm{C} 01$

[35] See Supplemental Material at http://link.aps.org/ supplemental/10.1103/PhysRevD.105.043018 for animations of the temporal evolution of survival probabilities.

[36] M.-R. Wu and I. Tamborra, Fast neutrino conversions: Ubiquitous in compact binary merger remnants, Phys. Rev. D 95, 103007 (2017).

[37] L. Johns, H. Nagakura, G. M. Fuller, and A. Burrows, Neutrino oscillations in supernovae: Angular moments and fast instabilities, Phys. Rev. D 101, 043009 (2020).

[38] M. Chakraborty and S. Chakraborty, Three flavor neutrino conversions in supernovae: slow \& fast instabilities, J. Cosmol. Astropart. Phys. 01 (2020) 005.

[39] F. Capozzi, M. Chakraborty, S. Chakraborty, and M. Sen, Fast Flavor Conversions in Supernovae: The Rise of $\mathrm{Mu}-$ Tau Neutrinos, Phys. Rev. Lett. 125, 251801 (2020). 